\title{
Clustering in Ad Hoc Personal Network Formation
}

\author{
Yanying Gu, Weidong Lu, R.V. Prasad, and Ignas Niemegeers \\ Center for Wireless and Personal Communications (CWPC), \\ Delft University of Technology, Delft, The Netherlands \\ $\{y \cdot g u, w .1 u, v p r a s a d, i . n i e m e g e e r s\} @ e w i . t u d e l f t . n l$
}

\begin{abstract}
Personal Network (PN) is a user-centric concept to realize the interconnection of the users' various devices and networks, such as home networks, corporate networks and vehicle area networks, at any time and any place. These networks may be geographically separated from each other and are usually organized in an ad hoc fashion, where the devices in one network can share content, data, applications and resources with each other, and communicate with the outside world. Clustering for personal networks intends to organize these ad-hoc networked heterogeneous devices into hierarchical clusters. A new clustering scheme, Personal Network Clustering Protocol (PNCP), is proposed for PNs and compared with clustering algorithms in the literature in terms of various performance metrics. PNCP performs the cluster formation based on heterogeneity of PNs in a distributed way, forms clusters with a moderate number of nodes, limits the cluster radius to $k$-hops, decreases the change in the cluster composition, extends the average membership time in the clusters providing stability and generates less overhead traffic. Though it was aimed for PNs, this protocol can also be used for any other heterogeneous ad hoc network for clustering.
\end{abstract}

Keywords: Personal network, Clustering, Ad hoc network, Personal Network Clustering Protocol.

\section{Introduction}

Although several existing technologies can offer a part of the solutions to communication needs of a person such as WPAN, WLAN, the Internet, and UMTS networks etc., little has been done to integrate these technologies and emerging ones to meet the future demands of a user. Thus the necessity is to combine all the devices of a user and networks into one single network which is transparent to the user. A Personal Network (PN) [1] supports a user's need to have access to his personal and public services with his devices. A PN covers all types of devices that can be used by a person in different places by using the service of interconnecting.

From the point of view of a user, the devices belonging to the owner of that PN are called personal devices (or personal nodes) [1], as shown in Fig. 1. The nodes having full networking functionalities such as packet forwarding, mobility management and computational capacities are full function nodes (FFN) [2]; the other nodes are called 
reduced function node (RFN) [2]. For example, a RFN does not forward packets or only forward (signaling) packets with high QoS requirements or packet catering to special purposes. Moreover, to form a secure private network and to achieve efficient communication between many devices of a PN, personal devices are naturally organized in groups, in which personal nodes can share their content, resources, etc. and cooperate with each other to support all the activities of the owner.

When personal devices are spread geographically, clustering is a way to organize the devices of a PN in the close vicinity of each other into hierarchical groups, which can facilitate efficient routing, addressing and self-organization of a PN. The clustered architecture guarantees a better performance in an ad hoc mobile network with a large number of mobile terminals [3]. Based on the properties of PNs, there are some specific requirements for the clustering scheme in PNs: (a) it must be distributed and self-organized [1]; (b) considering the privacy and the security in PNs, personal nodes and foreign nodes need to be included in personal clusters and foreign clusters [2]; (c) the proposed clustering scheme should give solutions for both FFN and RFN [2]; (d) since personal nodes are usually moving together in groups, the proposed clustering method should reduce the influence of the group mobility on the existing cluster relationship [5]; (e) the heterogeneity of personal nodes should be considered in the selection of a cluster head in each cluster [2]; (f) the life-time of the personal clusters should be long so that the influence of re-clustering on the applications and in turn the traffic load could be reduced [3]; (g) the cluster radius and the number of nodes in each personal cluster should be controlled in order to offer efficiency in PNs [3].

To efficiently manage a PN, we introduce a clustering scheme called Personal Network Clustering Protocol (PNCP) to generate a cluster structure to bridge different technologies and offer a homogeneous and a transparent view of a PN to the user. PNCP considers the heterogeneity of PNs in cluster formation in a distributed and self-organized way, forms clusters with a moderate number of nodes, limits the maximum number of hops of cluster radius [3], results in less variations cluster dynamics change, longer average membership time and less overhead compared to other clustering schemes.

The remainder of this paper is organized as follows. In Section 2, some clustering algorithms reported in the literature are presented. In Section 3, the descriptions of the proposed clustering protocol (PNCP) are highlighted. Section 4 offers explanations of various simulation results, which include the performance analysis and comparison between PNCP and other clustering algorithms. Finally, Section 5 summarizes our paper with conclusions, which include the contributions and performance improvements of PNCP.

\section{Related Work}

Many papers focused on presenting effective and efficient clustering schemes [3] for ad hoc networks. In the Lowest-ID clustering algorithm [6], the node with lowest ID in its neighborhood acts as a cluster head $(\mathrm{CH})$. However, the node with a lower ID would stay as a $\mathrm{CH}$ for a long period of time, which may cause the node draining the battery quickly. Least Cluster head Change (LCC) algorithm [3] still selects the nodes with lower ID as CHs and reduces the frequent re-clustering in Lowest_ID. Re-clustering is 
event-driven when two $\mathrm{CHs}$ move into the radio range of each other, one of them gives up the cluster head role. LCC significantly improves cluster stability by relinquishing the requirement that a $\mathrm{CH}$ should always bear some specific attributes in its local area.

Connectivity-based $k$-hop clustering (Highest_C) algorithm [8] is proposed to use connectivity as a primary and lower ID as a secondary criterion in selecting cluster heads. It generalizes the connectivity to count all $k$-hop neighbors of the given node. However, Highest_C does not offer a stable cluster structure, because topology changes may dramatically influence nodes' degree or connectivity.

Ohta et al, [9] have proposed a clustering scheme, which controls the number of nodes in each cluster between an upper and a lower bound. However, it results in frequently cluster division and merging. Mobility based clustering (MOBIC) introduces a novel relative mobility metric to offer a more stable cluster structure for mobile ad hoc networks [5]. MOBIC is feasible and effective for ad hoc networks with group mobility behavior.

In the On Demand Weighted Clustering Algorithm [11], a combined-metric factor is calculated to select a $\mathrm{CH}$. The combined metric considers node degree, node distance, absolute speed and battery power. However, the relative mobility should be used instead of absolute mobility as a metric in the $\mathrm{CH}$ selection [5].

Different clustering approaches typically focus on one specific performance metric. However, the requirements of a PN clustering scheme explained in Section 1 have not been taken into account at the same time in the above algorithms. PNCP incorporates all the aforementioned requirements and fulfill these to the extent possible.

\section{Personal Network Clustering Protocol (PNCP)}

Personal Network Clustering Protocol (PNCP) enables distributed and self-organized cluster formation and maintenance without any information, such as location, offered by other systems, such as GPS. Fig.1 gives an overview of the cluster structure in PNs. PNCP organizes personal nodes [1] to form personal clusters (Requirement $b$ in Section 1). Master Node (MN) [2] is a new term introduced in PN, which provides many PN specific functions as well as all the functionalities of a cluster head $(\mathrm{CH})$ of a personal cluster. In this paper, we only discuss $\mathrm{MN}$ functionalities as a $\mathrm{CH}$. In order to comply with $\mathrm{PN}$ terminology, we refer to $\mathrm{CH}$ as $\mathrm{MN}$, and use these terms interchangeably in the rest of this paper. The selection of $\mathrm{MN}$ is based on the node's capability to act as a MN, which is described by Node Capacity (NC) and Mobility $(M)$
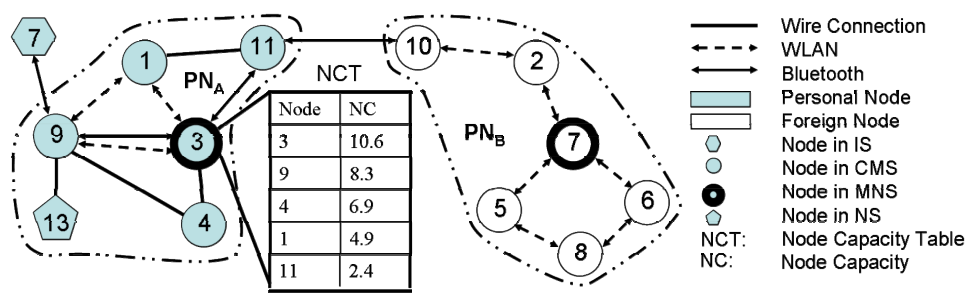

Fig. 1. Personal network clustering protocol 
(Requirement e). We describe the protocol using a model in which we associate each node with a state, which is explained in Fig. 1. Master Node State (MNS) and Cluster Member State (CMS) are defined for the MNs and CMs [2]. Initial State (IS) is taken by a personal node not belonging to any cluster, such as node 7 in Fig. 1. Further, we define a Non-clustered State (NS) for two kinds of personal nodes: (i) a RFN node chooses a $\mathrm{CM}$ as its gateway to access a personal cluster (Requirement $c$ ); and (ii) a CM may temporarily move to $k+1$ hops away from the master node and the node can set another CMS node in the same cluster as a gateway to extend its membership of the cluster (Requirement $f$ ) [10].

\subsection{Node Capacity and Mobility}

Many earlier clustering schemes perform cluster formation based on a single metric, for example, node identity, connectivity, mobility, etc. [3]. The On Demand Weighted Clustering Algorithm [11] tried to use a combined metric to achieve overall improvement. $N C$ explained in Eq. (1) is also a combined metric and proposed to consider the heterogeneous properties of personal nodes in PNs for the role of a MN. NC considers Connectivity $(C)$ and available Resources $(R)$ of a personal node, where $C$ indicates the influence of neighbors on the $N C$ and $R$ represents the internal capacity of the node.

$$
N C=\alpha_{1} * C+\alpha_{2} * R
$$

where $\alpha_{1}$ and $\alpha_{2}$ are the weight factors of $C$ and $R$. Geng Chen [8] has used connectivity as the criteria to select a $\mathrm{CH}$, where each neighbor in $k$-hop neighborhood make an equal contribution to $C$. Due to the location and capability difference of these neighbors, they may have different impacts on $C$. So we define connectivity $C$ in Eq. (2)

$$
C=\sum_{i=1}^{K} \frac{1}{i} \sum_{j}^{\mathrm{Ni}(\mathrm{v})}\left(T_{j}-M_{\mathrm{j}}\right)
$$

where $i$ is the $i^{\text {th }}$ neighborhood of node $v, k$ is the cluster radius in each cluster, $N i(v)$ denotes the nodes in the $i^{\text {th }}$ neighborhood, $T_{j}$ and $M_{j}$ are the transmission range and the mobility of the $j^{\text {th }}$ node in the $i^{\text {th }}$ neighborhood. The calculation method of $M_{j}$ is the same as $M_{Y}$ in [5]. Because the contribution of a neighbor decreases as the hop distance increase, we divide the contribution of each neighbor by its hop distance. Further for each neighbor, when it has a higher value of mobility, it may leave the neighborhood easily; with a larger transmission range it is not easy for the node to leave the neighborhood. Thus we use the result of $T$ minus $M$ to indicate the heterogeneity of each neighbor.

For $R$, we take into account available memory $(A)$, battery power $(E)$ and CPU resources $(P)$. We calculate the available resources, $R_{i}$, for node $i$, in Eq. (3):

$$
R_{i}=\left(\chi_{1} * A_{i}+\chi_{2} * P_{i}\right) * E_{i}
$$

The parameters, $\chi_{1}$ and $\chi_{2}$ are the weight factors. Although a node may have more available memory and CPU resources, it still can not use these resources if it 
has a low level of battery power. Thus we multiply the sum of the available memory and the CPU resources by $E_{i}$ to indicate the decisive role of $E_{i}$ in the calculating of $R$.

For Mobility, we use relative mobility $\left(M_{i}^{r e l}(j)\right)$ instead of absolute mobility, which has been proposed in [5]. And the Mobility $\left(M_{i}^{j}\right)$ of a personal node $j$ at a node $i$ is the absolute value of $M_{i}^{\text {rel }}(j)$, so the mobility metric is always positive. Thus a IS node chooses a $\mathrm{MN}$ with a lower relative mobility $(M)$ and joins the cluster to act as a $\mathrm{CM}$; and a IS node with highest ability $(N C)$ can change to $\mathrm{MN}$, which improves the stability of the formed cluster structure by the effort made by both MNs and CMs. NC and $M$ are used extensively in the cluster formation and maintenance algorithm, which will be explained in the following sections.

\subsection{Cluster Formation}

The actions taken in the cluster formation algorithm are explained as follows:

1: A IS node discovers its personal neighbors, calculates its $N C$ and relative $M$, and saves its $N C$ and all its personal neighbors' $N C$ in node capacity table (NCT) as shown in Fig. 1.

2: The IS node, as shown by node 7 in Fig. 1 , looks for a MN with lower $M$ from the existing neighbor personal clusters.

3: The IS node unicasts a hello message as a request to the MN as node 3 in Fig. 1 to apply to join the cluster.

4: The MN controlling the number of nodes in the cluster sends a response message as a decision to add the IS node in the personal cluster.

5: If step 1 to 3 are not successful, the IS node with highest $N C$ amongst all the IS neighbors changes to MNS, which is shown as node 3 in Fig. 1.

6: Other IS nodes follow Step 1 until either joining a cluster or changing to MNS.

From the above steps, our cluster formation algorithms has these contributions: (1) the cluster formation is only performed among personal nodes as indicated by Step 1, which meets the Requirement $a$; (2) personal nodes with similar mobility pattern in the neighborhoods join to the same cluster, because personal nodes choose the MN with the lower $M$ and join the cluster as described by Step 2, which meets the Requirement $d$; (3) existing cluster structure is not influenced by the new nodes starting in PN, because they always first try to join the existing personal clusters instead of forming new clusters as described in Step 2 and 3, which meets the Requirement f; (4) the MN controls the number of nodes in the cluster below $U$ by sending acceptation messages to the IS nodes trying to join the cluster as stated in Step 4, which meets the Requirement $g$; (5) the node with highest $N C$ in the local neighborhood can declare itself as a MN as node 3 in Fig. 1 as described in Step 5, so the MN selection considers the heterogeneity of nodes and meets the Requirement $e$.

\subsection{Cluster Maintenance}

The aims of the cluster maintenance algorithm are to increase the stability of the existing cluster structure in case of mobility in PN (Requirement $f$ ) and to control the 
number of nodes $(n)$ in each cluster (Requirement $g$ ) [3], [9]. To improve the stability of the cluster structure, re-clustering is carried out only in the following two cases:

1. A MN does not have enough resource (e.g. power) to act as a MN.

2. To control the $n$ in each cluster, two clusters may merge into one cluster to increase the $n$ in the cluster by "Cluster Merge" [9].

MNs are responsible for controlling $n$ in each personal cluster. To reduce frequent cluster division and merging [9], there is no cluster division in our algorithm. Each IS node sends a request to apply to join a cluster and the $\mathrm{MN}$ will decide whether to accept it or not to control $n$ below $U$. And to avoid small clusters, MNs checks $n$ periodically to perform "Cluster Merge". Both cluster radius [3] $(<=k)$ and cluster size $(L<n<U)$ [9] are considered as requirements in "Cluster Merge". Based on these requirements, a $\mathrm{MN}$ (requesting $\mathrm{MN}$ ) having a lower $n$ selects a neighbor MN (selected $\mathrm{MN}$ ) and sends a request to it. Considering these requirements, the selected MN may accept the requesting MN by sending a response. Then the requesting MN changes to CMS and all the CMs in the personal cluster join the selected cluster.

\section{Performance Evaluation}

PNCP is implemented in ns-2 [12] and is compared with other clustering algorithms for performance evaluation. The parameters of the simulation environments are set as shown in Table 1. Some performance metrics are proposed as the basis for evaluating the performance of the proposed clustering protocol: Average Master Node Time $(\mathrm{AMNT})$, which is defined as the average period of time during which a master node plays a central controller role continuously; Average Cluster Member Time (ACMT); Average Cluster Maintenance Load (ACML).

Table 1. Common set-up values for the simulations

\begin{tabular}{llll}
\hline Properties & Value & Properties & Value \\
\hline Terrain Dimension & $100 \mathrm{~m}^{*} 100 \mathrm{~m}$ & Hello Interval & $1 \mathrm{~s}$ \\
Simulation Time & $100 \mathrm{~s}$ & Cluster Size Upper Limit $(N)$ & 30 nodes \\
MAC Layer Type & 802.11 & Cluster Size Lower Limit $(L)$ & 5 nodes \\
Weight Factor $\alpha_{l}, \alpha_{2}, \chi_{l}$ and $\chi_{2}$ & 1 & Cluster Radius $(k)$ & 2 hops \\
Transmission range & $15 \mathrm{~m}$ & & \\
\hline
\end{tabular}

The experiments are carried out to compare Highest_C, LCC, Lowest_ID and the proposed PNCP. Based on Fig. 2 (a), (b) and Fig. 3 (a), (b), PNCP has the highest AMNT and ACMT in all situations, which means that PNCP can offer a more stable cluster structure from the $\mathrm{MN}$ and $\mathrm{CM}$ perspective. Using $N C$ gives this performance improvement, because the calculation of $C$ considers the different contribution of each neighbor. Moreover, the power level $(E)$ plays a decisive role on the other kinds of available resources. 


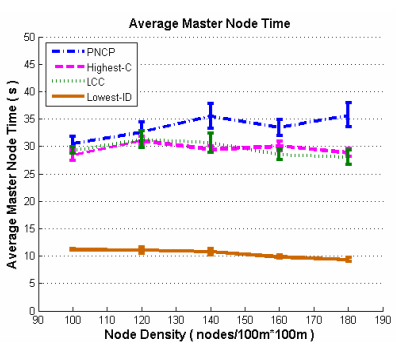

(a)

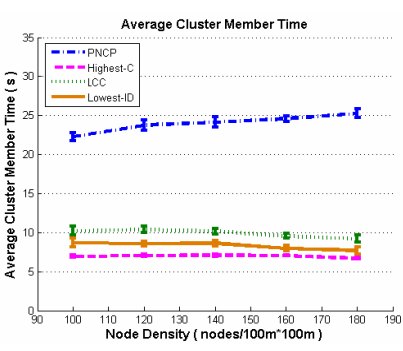

(b)

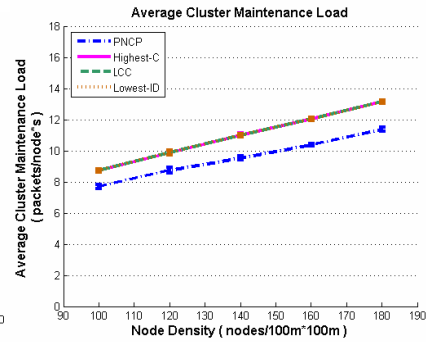

(c)

Fig. 2. Simulation results vs. node density with maximum speed $3 \mathrm{~m} / \mathrm{s}$

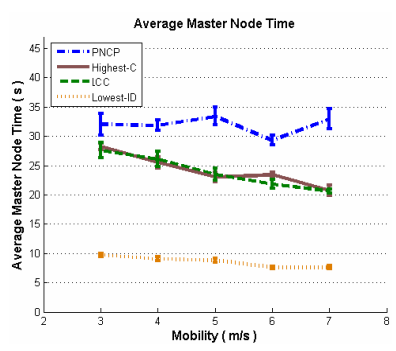

(a)

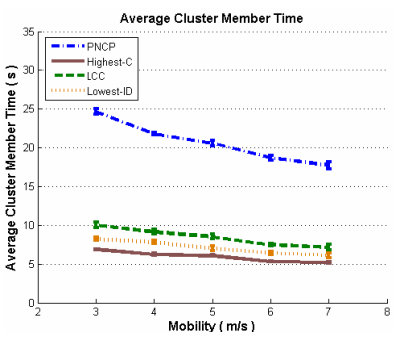

(b)

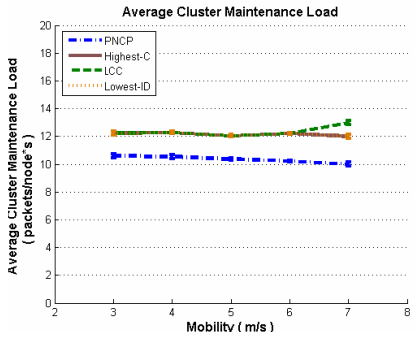

(c)

Fig. 3. Simulation results vs. node mobility with 90 nodes

From Fig. 2 (c) and Fig. 3 (c), PNCP has a lower ACML than the other clustering algorithms. The main reason is that the non-clustered state (NS) for a personal node to attach to its original personal cluster through a gateway. A NS node unicasts member messages to its one-hop gateway, which generates less traffic than a normal node that broadcasts member messages to personal neighbor nodes within $k$-hops.

Based on the simulations with different numbers of nodes and node mobility, PNCP provides some performance improvements: decreasing re-clustering, extending the average membership in the clusters, having a moderate number of nodes in each cluster, limiting cluster radius and generating less overhead. However, the study still has some limitations. Firstly, because of the limitation of ns-2, the simulation scenarios are not typical for PNs, however it is used here to study any ad hoc network simulation. Secondly, only fully functional nodes having complete networking functionality are considered in the simulation.

\section{Conclusion}

Clustering is the way ahead for organizing the heterogeneous nodes of PNs and in fact any ad hoc networks. We introduced a Personal Network Clustering Protocol (PNCP) for the distributed cluster formation and maintenance that are highly adaptive to the characteristics of PNs and overcome limitations of its predecessors in the literature. 
PNCP meets the PN clustering requirements as explained in Section 1 and can form a more stable cluster structure with a lower overhead in most of the cases. In addition, PNCP considers heterogeneity in PNs, forms clusters with moderate number of nodes, and limits the cluster radius within $k$-hops. PNCP is a generic protocol which can be used to form clusters in any ad hoc networks. Future research in PNs can benefit from the cluster structure provided by PNCP. A flexible routing protocol exploiting the hierarchical structure formed by PNCP is needed to provide a better performance for PNs. Furthermore, a security method for PN clustering should be specified and analyzed along with the clustering. The field of PNs is rapidly growing and changing, while there are still many challenges that need to be met.

\section{References}

1. Ignas G. M. M. Niemegeers, Sonia M. Heemstra de Groot: Research issues in ad-hoc distributed personal networking. Wireless Personal Communications: An International Journal, Volume: 26, Issue: 2-3, Kluwer Academic Publishers (2003) 149-167

2. W. Lu (ed.): D1.2 Initial Architecture of Personal Networks. IOP GenCom Project QoS for Personal Network at Home Deliverable (2005)

3. J. Y. Yu and P. H. J. Chomg: A Survey of Clustering Schemes for Mobile Ad Hoc Networks. IEEE Communications Surveys and Tutorials, Vol. 7, No. 1 (2005) 32-48

4. M.R. Pearlman and Z.J. Haas: Determining the Optimal Configuration for the Zone Routing Protocol. IEEE JSAC, vol 17 (1999) 395-414

5. P. Basu, N. Khan, and T. D. C. Little: A Mobility Based Metric for Clustering in Mobile Ad Hoc Networks. In Proc. of Workshop on Wireless Networks and Mobile Computing (2001)

6. M. Gerla and J. T.C. Tai: Multi cluster, Mobile Multimedia Radio Networks. Wireless Networks 1 (1995) 255-265

7. C. C. Chiang, H. K. Wu, W. Liu and M. Gerla: Routing in Clustered Multihop, Mobile Wireless Networks with Fading Channel. In Proc. IEEE SICN (1996) 197-211

8. F.G. Gonzalez, J.S. Gonzalez: Connectivity based k-hop clustering in wireless networks. Proc. $35^{\text {th }}$ Hawaii International Conference on System Sciences (2002)

9. T. Ohta, N. Murakami, R. Oda, and Y. Kakuda: An Improved Autonomous Clustering Scheme for Highly Mobile Large Ad Hoc Networks. Workshops on AHSP (2005) 655-660

10. J. Y. Yu, and P. H. J. Chong: 3hBAC (3-hop between adjacent clusterheads): A novel nonoverlapping clustering algorithm for mobile ad hoc networks. Proc-IEEE PacRim 03, Canada (2003)

11. M. Chatterjee, Sajal K, D. Turgut: An on-demand Weighted Clustering Algorithm (WCA) for Ad hoc networks. Proceedings of IEEE GLOBECOM (2000) 1697 - 1701

12. Kevin Fall and Kannan Varadhan: The ns Manual. http://www.isi.edu/nsnam/ns/doc.pdf (2005) 